\title{
Medical Implications of Antimicrobial Coating Polymers- Organosilicon Quaternary Ammonium Chloride
}

Yuen John WM* and Yung Jolene YK

School of Nursing, The Hong Kong Polytechnic University, Hung Hom, Kowloon, Hong Kong

\begin{abstract}
Hospital-Associated Infections ( $\mathrm{HAI})$ are commonly caused by the invasive device and prosthesis implanted in the body. The practice of coating antimicrobial agents on biomedical surfaces or modifying the composite resin with such agents has been shown effective in reducing the incidence of HAI. Quaternary Ammonium Chloride (QAC) salts and the Organosilicon derivatives (OrganoSiQAC) are surface active to serve these applications. Clinically, Benzalkonium chloride (BAC) is effective against a board range of microorganisms. However, it has been recognized as the source of several hospital outbreaks due to contamination with gram-negative bacteria. Safety aspects such as potential toxicities and in vivo efficacies were poorly defined. On the other hand, physical antimicrobial polymers formed by OrganoSiQAC compounds were found to be chemically stable and nonleachable from the bonded surface, whereas the biocidal effects were exerted by the end satellite QAC groups. Recent studies have also reported the application of such bioactive films on animated surfaces such as skin and mucosal lining. This opens the future perspective with multiple applications in infection control, in the regards of reduce use of antibiotics, treatment alternatives for multiple antibiotic resistance, blocking the route of transmission of specific organisms in clinical and community settings.
\end{abstract}

Keywords: Ammonium chloride; OrganoSiQAC compounds; Antibiotic resistance

\section{Introduction}

Quaternary ammonium compounds (Quats) especially, the chloride salts are extensively used for clinical purposes such as preoperative disinfection of unbroken skin, application to mucous membranes, and disinfection of noncritical surfaces [1]. The cationic properties of Quats contribute to a variety of applications as disinfectants, antiseptics, herbicides, spermicides, detergents, and sanitizing agents [2]. Organosilicon Quaternary Ammonium Chloride (OrganoSiQAC) salts are cationic polymeric materials that typically contain a Quaternary Ammonium Chloride (QAC) end group in the organosilicon architecture. Such organosilicon-substituted amines are well known active surfactant-mediated agents that are able to cause polymerization immediately after contacting any surface. Organosilicon chemistry is the designated field of science studying the properties and reactivity of all organic compounds containing carbon-silicon ( $\mathrm{Si}-\mathrm{C}$ ) bonds. In this mini-review, the authors are particularly interested in exploring two relative Quats - QAC and Organiosilicon; briefly discuss their chemistry and synthesis, utilization in healthcare as antimicrobial agents, safety issues, major shortcomings and future perspective.

\section{The Chemistry of Quats and OrganoSiQAC}

Quats share a common tetra alkyl ammonium structure of $\mathrm{N}^{+} 4 \mathrm{xR}$ where a nitrogen $\left(\mathrm{N}^{+}\right)$atom is directly linked to four alkyl groups $(\mathrm{R})$, forming positively charged polyatomic ions with a variety of complexity [3]. In the process of quaternization, a tertiary amine can be converted to Quats by alkylation, and typically one of the alkyl groups is larger than the others [4]. Benzalkonium Chloride (BAC), the most widely used QAC-based antiseptic in healthcare, is synthesized from the longchain alkyldimethylamine and benzyl chloride:

$$
\mathrm{CH}_{3}\left(\mathrm{CH}_{2}\right)_{\mathrm{n}} \mathrm{N}\left(\mathrm{CH}_{3}\right)_{2}+\mathrm{ClCH}_{2} \mathrm{C}_{6} \mathrm{H}_{5} \rightarrow\left[\mathrm{CH}_{3}\left(\mathrm{CH}_{2}\right)_{\mathrm{n}} \mathrm{N}\left(\mathrm{CH}_{3}\right)_{2} \mathrm{CH}_{2} \mathrm{C}_{6} \mathrm{H}_{5}\right]^{+} \mathrm{Cl}^{-}
$$

Besides BAC, other antimicrobial QAC compounds, such as alkyldimethylbenzylammonium chloride and didecyldimethylammonium chloride, are also used as active antimicrobial agents.

Silicon $(\mathrm{Si})$, on the other hand is tetravalent and tetrahedral that allows the formation of organosilicon amines with basic structure of
$\mathrm{Si} \cdot 4 \mathrm{xR}$, whereas OrganoSiQAC contains typically a QAC substitute as one of the alkyl groups [5]. When compared with the carbon-carbon (C-C) bonds of hydrocarbons, Si-C bonds are more readily to be broken because of the weaker bond dissociation energy ( $\mathrm{Si} 451 \mathrm{~kJ} /$ mol vs C $607 \mathrm{~kJ} / \mathrm{mol}$ ). The greater electronegativity (C 2.55 vs Si 1.90 ) of carbon atom contributes also to the polarization of Si towards the carbon. Compare with Quats and QAC which are entirely hydrocarbon in nature, the cationic OrganoSiQAC molecules have lower micelle concentrations and provide lower surface tension due to the presence of silicon in their hydrophobic groups. Despite organosilicons are highly hydrophobic, quaternization could further increase the water solubility of the compound. The synthesis of OrganoSiQAC involves multiple steps based on either one of the two catalytic methods: (1) the formation of tertiary amino silicon by $\mathrm{Si}-\mathrm{H}$ addition reaction, followed by reaction with haloalkane; and (2) the Menschutkin reaction between halogenoalkyl silicone unit and tertiary amines. Recently, Li et al. [6] has described a one-step Menschutkin reaction without catalyst and successfully synthesized two OrganoSiQAC molecules, namely Diethyl-benzyl-[3-methyldimethoxyl]silpropyl ammonium chloride (DEBSAC) and Trimethyl-[3-methyldimethoxyl]silpropyl ammonium chloride (TMSAC) as shown in Table 1. Both products were surface active and antibacterial against Escherichia coli (E. coli) (Table 1).

\section{Utilization of QAC as Antimicrobial Surfactant}

Hospital-Associated Infections (HAI) are commonly caused by the invasive device and prosthesis implanted in the body. The practice of coating antimicrobial agents on biomedical surfaces or modifying the composite resin with such agents has been shown effective in reducing

*Corresponding author: Dr. John WM Yuen, School of Nursing, the Hong Kong Polytechnic University, Yuk Choi Road, Hung Hom, Kowloon, Hong Kong Special Administrative Region, Hong Kong, Tel: +852-2766-4130; Fax: +852-2364-9663; E-mail: john.yuen@polyu.edu.hk

Received June 21, 2013; Accepted July 27, 2013; Published August 02, 2013

Citation: Yuen John WM, Yung Jolene YK (2013) Medical Implications of Antimicrobial Coating Polymers- Organosilicon Quaternary Ammonium Chloride. Mod Chem appl 1: 107. doi:10.4172/2329-6798.1000107

Copyright: $\odot 2013$ Yuen John WM, et al. This is an open-access article distributed under the terms of the Creative Commons Attribution License, which permits unrestricted use, distribution, and reproduction in any medium, provided the original author and source are credited. 
the incidence of HAI. This is attributed by the surfactant property of QAC and OrganoSiQAC compounds, which allows the absorption of the compound molecules to form a protective coating and the reaction with the surfaces to form a new antimicrobial film, respectively. The underlying anti-infective mechanism of such surfactant activity lies on the prevention of microorganism adherence to resist biofilm formation $[7,8]$. 'Powder test' has confirmed the theory of absorption of cations to prepare antimicrobial surfaces, especially on fibers and textiles [2]. Cations alone were discovered to be responsible for antimicrobial behavior [2].

Studies have demonstrated that colonization of a range of pathogenic organisms, including gram- positive and negative species as well as Candida albicans were inhibited by coating the surface of central venous catheters with BAC $[9,10]$. BAC-modified orthodontic composite resin has shown to exert inhibitory activity against Streptococcus mutants (common cause of dental caries), without affecting the bond strengths of the material [11]. However, such antimicrobial properties on surfaces were merely evidenced by in vitro experiments [9-12]. The in vivo effectiveness is doubtful, since in the study of Imbert et al. [10], the inhibition of $C$. albicans adherence exerted by BAC has become ineffective when the plastic surface was layered with an extracellular matrix gel [10]. Nonetheless, numerous QAC-based surfactants are nowadays developed into nonascale that could also serve as vesicles for drugs. A successful case was reported by Matl et al. [12] that hydrophilic antibiotic drugs were bond together with QAC compounds onto the lipophilic surfaces of Polytetrafluoroethylene (PTFE) prostheses, where antibiotics were being delivered in vivo after the prosthetic implantation.

Despite it is already the most studied QAC compound, since 1994, BAC has been classified by the U.S. Food and Drug Administration as a Category IIISE active antiseptic, which means data is insufficient to classify as safe and effective [13]. Epidemiologically, several hospital infection outbreaks were associated with contamination of BAC solutions with bacteria, especially the gram negative species [14]. Several organisms, mainly the glucose non-fermentative bacillus including Pseudomonas aeruginosa (an important causative agent of nosocomial infections), were viable to grow in the diluted BAC solution and $0.02 \%$ BAC-soaked cotton balls [15]. More specifically, BAC solutions contaminated with Serratia marcescens have been associated with reported cases of lethal infections such as meningitis [16] and septic arthritis [17]. Nakashima et al. [18] have reported the survival ability of Serratia marcescens in BAC solutions. Apart from the contamination issues, the biocidal nature of QAC compounds has also drawn the concerns about resistance development [19] and potential toxicities [20]. Several bacterial isolates, including Staphylococcus aureus, displaying decreased susceptibility to QACs have been reported [21]. Particularly, QAC compounds of lower molecular weight are gradually released from the bound surface, leading to the loss of antimicrobial activity over time while such leaching particles could also be toxic in vivo if it is not properly controlled [20].

\section{The Era of OrganoSiQAC and Future Perspective}

In general, since there is a new physical layer formed on the surface, polymeric biocides are known to have certain advantages: (1) stable and without releasing low molecular weight toxic products to the environment; (2) no problem of residual toxicity; (3) durable and sustainable antimicrobial activity; and (4) common bacterial strains do not appear to develop resistance [22]. Thermal stable polysiloxane polymers containing $\mathrm{N}, \mathrm{N}$-dialkylimidazolium halide groups are very potent against a broad spectrum of bacteria [22,23]. A research team led by Walters et al. [24] has first demonstrated the formation of a waterproof firm with an OrganoSiQAC, known as Quat-Silsesquioxane (3-(trimethoxysilyl)-propyldimethyloctadecyl ammonium chloride) through hydrolysis on glass surfaces. Quat-Silsesquioxane has been tested in mammal animals and revealed its extremely low toxicities, in term of tetratogenicity and abnormal foetus development $[25,26]$. As shown in Figure 1, when immobilized on the reactive surface, the QAC compartment of Quat-Silsesquioxane acts as the functional group for microbicidal activities against representative species of alga [24], bacteria and fungi [23]. In late 90s, Saito and colleagues [27] developed a spray solution contains a mixture of Quat-Silsesquioxane and spherical silica particles, which improve the efficiency and quality of bioactive film, using a spray-dry procedure. A nonleachable adhesive coating was successfully polymerized on the oxidized silicone rubber surface upon silanization, and provided stable and long-lasting antimicrobial protection both in vitro and in rats in vivo [28]. Later on, the shortcomings of peeling off and be scraped off from the surface were addressed by the layer-by-layer self-assembly approach [29]. Multilayer coatings for medical implants supply the dual functions to prevent bacterial attachment and to provide versatility of tunable release of therapeutic agents [30]. Recently, a new nanotechnology known as JUC (Brand name) was shown to be beneficial in preventing the growth of $E$. coli (a common cause of Urinary Tract Infection (UTI)) on the surface

\begin{tabular}{|c|c|c|c|}
\hline Compound & Reaction & Raw materials used & Reaction condition \\
\hline DEBSAC & 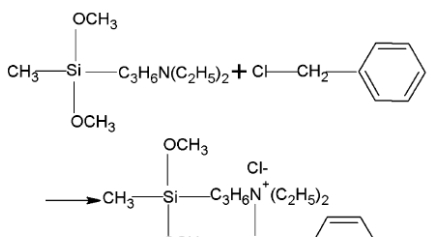 & $\begin{array}{l}\text { (1) } \mathrm{N}, \mathrm{N} \text {-diethyl-aminopropyl- } \\
\text { methyldimethozysilanes } \\
\text { (2) Benzyl chloride } \\
\text { Molar ratio }=1: 1.2\end{array}$ & Reflux at $80^{\circ} \mathrm{C}$ for 22 hours. \\
\hline TMSAC & $\underset{\mathrm{Cl}-\left(\mathrm{CH}_{2}\right)_{3}-\mathrm{Si}-\mathrm{CH}_{3}+\mathrm{N}\left(\mathrm{CH}_{3}\right)_{3}}{\mathrm{CH}_{3}} \stackrel{ }{\mathrm{OCH}_{3}}$ & $\begin{array}{l}\text { (1) trimethylammonium ethanol } \\
\text { solution }(30 \%) \\
\text { (2) } y \text {-chloropropylmethyl- } \\
\text { dimethoxysilane } \\
\text { Molar ratio }=1.3: 1\end{array}$ & $\begin{array}{c}\text { Stir the mixture at } 90^{\circ} \mathrm{C} \text { for } 12 \\
\text { hours. }\end{array}$ \\
\hline
\end{tabular}

Table 1: The one-step non-catalytic synthesis of two OrganoSiQAC compounds [6]. 
of siliconized latex urinary catheters; and this property was reported to reduce the incidence rate of catheter-associated UTI, from $13.04 \%$ to $4.52 \%$, in a randomized controlled clinical trial with 1,150 patients [31]. So far, the antimicrobial coatings discussed were all applied on inanimate surfaces, but could they be used on animate surfaces such as hands and skin? (Figure 1).

The JUC spray is marketed as a physical antimicrobial dressing for wound care, its formulation composed of $2 \%$ OrganoSiQAC as key ingredient, despite the exact OrganoSiQAC formulae has not been disclosed by the manufacturer. This forms a cutting-edge technology indicative to be applicable on skin and mucosal surfaces. The idea is simple enough underlying the principle that avoidance of microbial adherence on the surface and surrounding of wounds would prevent wound infection, and thus favor wound healing. At first, it was believed that microcidal effects of polymers formed by OrganoSiQAC compounds were proportionally correlated with the length of the carbon chain in the alkyl moieties and their abilities of polymerization [32]. Actually, the killing activities are dependent on the satellite end groups determined by the formation of unimolecular micelles as consequence of the polyoxazolines aggregation behavior, instead of the polymeric chain lengths [33]. Take JUC as example, immediately after contacting any surfaces, an invisible antimicrobial layer with dual overlaying structure: the bonded film and the positive charge film are produced. The bonded film aims to secure the adhesion to the surface, whereas positive charge film contains polycations bearing considerable positive charge may destructively interact through electrostatic forces with the negatively charged bacteria walls and membranes leading to the killing effects (Figure 2) [22,34].

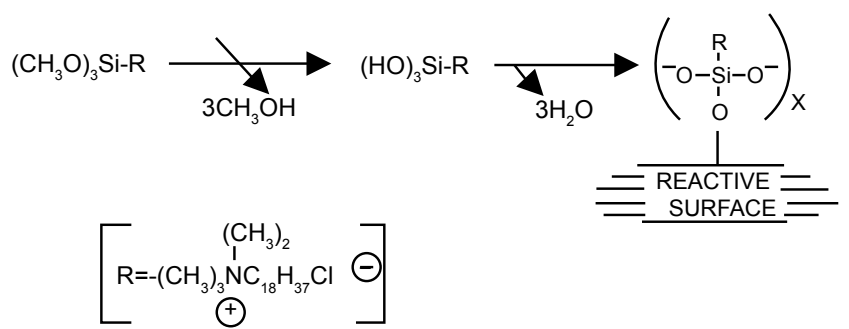

Figure 1: Diagram adopted from [23,24] displaying the hydrolysis reaction of Quat-Silsesquioxane on glass surface. Microcidal activity is exerted by the R group exposed at the bound surface.

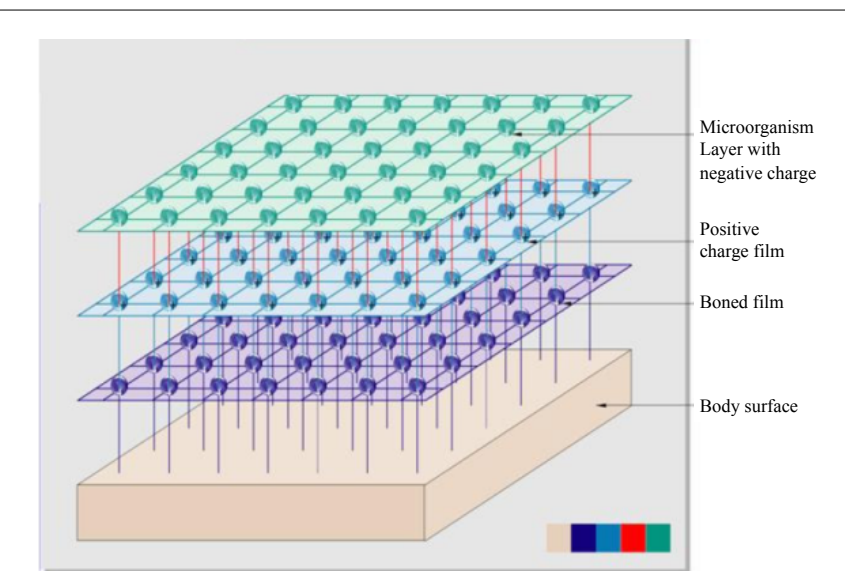

Figure 2: Diagram adopted from [34] showing the dual layers form by JUC spray to exhibit antimicrobial function.
Clinically, several studies conducted in Mainland China have already been demonstrated the effectiveness of JUC spray on preventing post-operative infection and reducing wound healing time in patients who received different surgical procedures, such as circumcision in males [35] and tumor removal of oral cancer patients [34]. In neck and head cancer patients who are receiving radiation therapy, JUC when applied at the onset of acute dermatitis, was shown to relieve the itching and pain sensation, promote wound healing, and reduce the incidence of secondary infection by $13 \%$ [36]. In another study conducted with patients in Intensive Care Unit (ICU) of a major Hong Kong hospital, the incidence rate of ventilator-associated pneumonia was significantly reduced from $54.2 \%$ to $8.4 \%$ after the application of JUC to the oral and nasal cavity [37]. Furthermore, a case of scalp abscess infected with methicillin-resistant Staphylococcus aureus (MRSA) was successfully treated solely with JUC spray without other form of treatment including antibiotics [38]. This reported case supported not only future investigation regarding the therapeutic roles of JUC and other OrganoSiQAC, but also suggested novel treatment alternative for multiple drugs resistance the potential reduction of antibiotics usage. In our laboratory, the efficacy of using JUC as a long-lasting hygienic handrub product was evaluated according to the requirements of European Standard, and another study was underway to investigate the effect of JUC in blocking MRSA transmission in a hospital ward environment. The OrganoSiQAC-based antimicrobial nanotechnology could be the trend of 'modern tools' to address important issues of infection control: reduce use of antibiotics, treatment alternatives for multiple antibiotics resistance, blocking the route of transmission of specific organisms in clinical and community settings.

\section{Conclusion}

In summary, the chemistry of QAC and OrganoSiQAC compounds has formed the fundamental concept about physical antimicrobial surface. The current body of literature identifies BAC and QuatSilsesquioxane as typical clinically used. Newly emerging OrganoSiQAC nanotechnology such as JUC may be the future direction of research and application.

\section{References}

1. Tischer M, Pradel G, Ohlsen K, Holzgrabe U (2012) Quaternary ammonium salts and their antimicrobial potential: targets or nonspecific interactions? ChemMedChem 7: 22-31.

2. Speier JL, Malek JR (1982) Destruction of microorganisms by contact with solid surfaces. Journal of Colloid and Interface Science 89: 68-76.

3. Alum A, Rashid A, Mobasher B, Abbaszadegan M (2008) Cement-based biocide coatings for controlling algal growth in water distibution canals. Cement and Concrete Composites 30: 839-847.

4. Kuca K, Kivala M, Dohnal V (2004) A general method for the quaternization of $\mathrm{N}, \mathrm{N}$-dimethylbenzylamines with long chain $\mathrm{n}$-alkylbromides. Journal of Applied Biomedicine 2: 195-198.

5. Soula G, Gerard Mignani M (1993) Organosilicon compounds. US Patent 5214176.s

6. Li J, Zhang Q, Wang Y, Zhang W, Li T (2012) Synthesis and properties of organosilicon quaternary salts surfactants. Journal of Surfactants and Detergents 15: 339-344.

7. Carmona-Ribeiro AM, de Melo Carrasco LD (2013) Cationic antimicrobial polymers and their assemblies. Int J Mol Sci 14: 9906-9946.

8. Kugel A, Stafslien S, Chisholm BJ (2011) Antimicrobial coatings produced by "tethering" biocides to the coating matrix: A comprehensive review. Progress in Organic Coatings 72: 222-252.

9. Tebbs SE, Elliott TS (1993) A novel antimicrobial central venous catheter impregnated with benzalkonium chloride. J Antimicrob Chemother 31: 261-271.

10. Imbert C, Lassy E, Daniault G, Jacquemin JL, Rodier MH (2003) Treatment of 
Citation: Yuen John WM, Yung Jolene YK (2013) Medical Implications of Antimicrobial Coating Polymers- Organosilicon Quaternary Ammonium Chloride. Mod Chem appl 1: 107. doi:10.4172/2329-6798.1000107

Page 4 of 4

plastic and extracellular matrix components with chlorhexidine or benzalkonium chloride: effect on Candida albicans adherence capacity in vitro. J Antimicrob Chemother 51: 281-287.

11. Sehgal V, Shetty VS, Mogra S, Bhat G, Eipe M, et al. (2007) Evaluation of antimicrobial and physical properties of orthodontic composite resin modified by addition of antimicrobial agents--an in-vitro study. Am J Orthod Dentofacial Orthop 131: $525-529$

12. Matl FD, Obermeier A, Repmann S, Friess W, Stemberger A, et al. (2008) New anti-infective coatings of medical implants. Antimicrob Agents Chemother 52: 1957-1963.

13. Boyce JM, Pittet D (2002) Guideline for Hand Hygiene in Health-Care Settings. Recommendations of the Healthcare Infection Control Practices Advisory Committee and the HICPAC/SHEA/APIC/IDSA Hand Hygiene Task Force. Society for Healthcare Epidemiology of America/Association for Professionals in Infection Control/Infectious Diseases Society of America. MMWR Recomm Rep 51: 1-45.

14. Weber DJ, Rutala WA, Sickbert-Bennett EE (2007) Outbreaks associated with contaminated antiseptics and disinfectants. Antimicrob Agents Chemother 51: 4217-4224.

15. Oie S, Kamiya A (1996) Microbial contamination of antiseptics and disinfectants. Am J Infect Control 24: 389-395.

16. Sautter RL, Mattman LH, Legaspi RC (1984) Serratia marcescens meningitis associated with a contaminated benzalkonium chloride solution. Infect Control 5: 223-225.

17. Nakashima AK, McCarthy MA, Martone WJ, Anderson RL (1987) Epidemic septic arthritis caused by Serratia marcescens and associated with a benzalkonium chloride antiseptic. J Clin Microbiol 25: 1014-1018.

18. Nakashima AK, Highsmith AK, Martone WJ (1987) Survival of Serratia marcescens in benzalkonium chloride and in multiple-dose medication vials: relationship to epidemic septic arthritis. J Clin Microbiol 25: 1019-1021.

19. Fraise AP (2002) Susceptibility of antibiotic-resistant cocci to biocides. J Appl Microbiol 92 Suppl: 158S-62S.

20. Beyth N, Yudovin-Farber I, Bahir R, Domb AJ, Weiss El (2006) Antibacteria activity of dental composites containing quaternary ammonium polyethylenimine nanoparticles against Streptococcus mutans. Biomaterials 27: 3995-4002.

21. Carson RT, Larson E, Levy SB, Marshall BM, Aiello AE (2008) Use of antibacterial consumer products containing quaternary ammonium compounds and drug resistance in the community. J Antimicrob Chemother 62: 1160-1162.

22. Mizerska U, Fortuniak W, Chojnowski J, Halasa R, Konopacka A, et al. (2009) Polysiloxane cationic biocides with imidazolium salt $(\mathrm{ImS})$ groups, synthesis and antibacterial properties. European Polymer Journal 45: 779-787.

23. Isquith AJ, Abbott EA, Walters PA (1972) Surface-bonded antimicrobial activity of an organosilicon quaternary ammonium chloride. Appl Microbiol 24: 859-863.
24. Walters PA, Abbott EA, Isquith AJ (1973) Algicidal activity of a surface-bonded organosilicon quaternary ammonium chloride. Appl Microbiol 25: 253-256.

25. Siddiqui WH, Malek JR, Stanton E, Hobbs EJ (1983) Percutaneous absorption of an antimicrobial organosilicon quarternary ammonium chloride in rabbits. $J$ Appl Toxicol 3: 146-149.

26. Siddiqui WH, York RG (1993) Quaternary silsesquioxane: a developmenta toxicity study in rats. Fundam Appl Toxicol 21: 66-70.

27. Saito T, Takatsuka T, Kato T, Ishihara K, Okuda K (1997) Adherence of ora streptococci to an immobilized antimicrobial agent. Arch Oral Biol 42: 539-545.

28. Gottenbos B, van der Mei HC, Klatter F, Nieuwenhuis P, Busscher HJ (2002) In vitro and in vivo antimicrobial activity of covalently coupled quaternary ammonium silane coatings on silicone rubber. Biomaterials 23: 1417-1423.

29. Wong SY, Li Q, Veselinovic J, Kim BS, Klibanov AM, et al. (2010) Bactericidal and virucidal ultrathin films assembled layer by layer from polycationic $\mathrm{N}$-alkylated polyethylenimines and polyanions. Biomaterials 31: 4079-4087.

30. Wong SY, Moskowitz JS, Veselinovic J, Rosario RA, Timachova K, et al. (2010) Dual functional polyelectrolyte multilayer coatings for implants: permanent microbicidal base with controlled release of therapeutic agents. J Am Chem Soc 132: $17840-17848$

31. He W, Wang D, Ye Z, Qian W, Tao Y, et al. (2012)Application of a nanotechnology antimicrobial spray to prevent lower urinary tract infection: a multicenter urology trial. J Transl Med 10 Suppl 1: S14.

32. Chen CZ, Cooper SL, Tan NCB (1999) Incorporation of dimethyldodecylammonium chloride functionalities onto poly(propylene imine) dendrimers significantly enhances their antibacterial properties. Chem Commun 1999: 1585-1586.

33. Munoz-Bonilla A, Fernandez-Garcia M (2012) Polymeric materials with antimicrobial activity. Progress in Polymer Science 37: 281-339.

34. Zeng Y, Deng R, Yeung BHS, Loo WTY, Cheung MNB, et al. (2008) Application of an antibacterial dressing spray in the prevention of post-operative infection in oral cancer patients: A phase 1 clinical trial. African Journal of Biotechnology 7: 3827-3831.

35. Chuan L, Weili Z, Zili H, Shengjie Y, Zuo L (2010) Clinical study of long-acting antibacterial material JUC for prevention of incicion infection in circrmcision. Journal of Chongqing Medical University 35: 1271-1273.

36. Xian-ming D, Zhen K, Jia-zhu W (2013) Prevention and treatment of acute radiodermatitis with JUC physical antimicrobial spray dressing. Journal of Dermatology and Venereology.

37. Li W, Ma X, Peng Y, Cao J, Loo WTY, et al. (2011) Application of a nanoantimicrobial film to prevent ventilator-associated pneumonia: A pilot study. African Journal of Biotechnology 10: 1926-1931.

38. Wan KA, Ng MY, Wong YT (2011) New horizon on community-acquired methicillin resistant Staphylococcus aureus (CA-MRSA) skin and soft tissue infection: nanotechnology antimicrobial spray. HK J Emerg Med 18: 432-436. 